Revue d'histoire de l'Amérique française

ZRS REVUE D.HISTOIRE DE L'AMÉRIQUE FRANÇAISE

\title{
Artilleurs canadiens-français dans la bataille d'Allemagne (novembre 1944 - mai 1945) (suite et fin)
}

\section{Jacques Gouin}

Volume 18, numéro 2, septembre 1964

URI : https://id.erudit.org/iderudit/302362ar

DOI : https://doi.org/10.7202/302362ar

Aller au sommaire du numéro

Éditeur(s)

Institut d'histoire de l'Amérique française

ISSN

0035-2357 (imprimé)

1492-1383 (numérique)

Découvrir la revue

Citer cet article

Gouin, J. (1964). Artilleurs canadiens-français dans la bataille d'Allemagne (novembre 1944 - mai 1945) (suite et fin). Revue d'histoire de l'Amérique française, 18(2), 233-251. https://doi.org/10.7202/302362ar d'utilisation que vous pouvez consulter en ligne. 


\title{
ARTILLEURS CANADIENS-FRANÇAIS DANS LA BATAILLE D'ALLEMAGNE *

\author{
(novembre 1944 - mai 1945)
}

$9 e$ article

\author{
DU RHIN À WILHEMSHAVEN
}

\section{INTERMÈDE EN HOLLANDE}

Le 11 mars, le Régiment apprenait qu'il allait retourner en Hollande, pour y être attaché au $1^{\text {er }}$ corps britannique. Bien qu'on l'ignorât à l'époque, il s'agissait d'un simple répit de dix jours entre deux batailles. L'avant-veille de son départ, il engageait encore des cibles en collaboration avec un observateur aérien. Celui-ci, satisfait de la réaction des canons, envoyait au Régiment le commentaire suivant: "Response excellent, shooting consistent, lot of damage caused ${ }^{1 "}$.

Le 13 mars, après avoir appris officiellement que le major Tremblay pouvait porter sa D.S.O. gagnée à Falaise, le Régiment partait pour sa nouvelle position. Dès le lendemain aprèsmidi, il effectuait un tir de contre-batterie à la demande du $1^{\text {er }}$ corps britannique, qui l'informait ensuite de se préparer à partir pour le secteur de Nimègue ${ }^{2}$. Croyant sans doute à la fin du rôle du Régiment dans la guerre, un de ses officiers écrivait ce qui suit le 14 mars :

... nous sommes rendus très loin du front. En effet, nous sommes de retour en Hollande pour un repos bien mérité... Je ne sais pas pour combien de temps nous serons ici, mais d'après notre com-

\footnotetext{
${ }^{*}$ Voir notre Revue, XVI: 240-253, 353-368, 536-548; XVII: 70-86, 244-257, 325-339, 504-530; XVIII: 85-111.

1 Journal de guerre du Régiment, XXXVI: 5.

2 Ibid., 6.
} 
mandant, il est fort probable que notre guerre à nous soit pratiquement terminée, du moins jusqu'au moment où il faudra traverser le Rhin, si jamais nous avons à le traverser. Mais il est possible que l'Allemagne jette bas les armes avant... Tant que les Allemands ne cèderont pas, ce sera la destruction méthodique et implacable. Il n'en tient qu'à eux de faire cesser ce carnage effroyable ${ }^{3}$.

Ce même jour, évoquant le champ de bataille quitté depuis peu, cet officier écrivait à un autre correspondant:

Je n'ai jamais vu de champ de bataille aussi sinistre que celui qui s'étendait de Nimègue jusqu'à Xanten au bord du Rhin ! Tout est détruit, et des cadavres d'Allemands et d'animaux jonchaient, pêle-mêle, les champs et les routes ${ }^{4}$.

\section{DILEMME CHEZ L'ENNEMI}

Vers cette époque, le haut commandement allemand étudiait l'alternative suivante: tenir sur l'Oder contre les Russes ou rejeter sur le Rhin les Occidentaux ${ }^{5}$. Or, ni l'une ni l'autre de ces solutions ne fut tentée, comme on le sait maintenant. Les armées allemandes étaient alors prises dans un gigantesque étau, et rien ne pouvait les sauver de l'écrasement total.

Le 16 mars, le Régiment était de retour dans la région de Nimègue, son quartier général régimentaire étant installé exactement au même endroit que le 8 février précédent, au moment de l'opération "Veritable". Le soir du 17, l'artillerie ennemie tirait sur les positions du Régiment ${ }^{6}$. Et, le lendemain, quelques avions ennemis en faisaient autant, sans toutefois causer aucun dommage ?

\section{OPERATION "BLOCKBUSTER II"}

Le 18 mars, à $0530 \mathrm{~h}$. se déclenchait l'opération "Blockbuster II", avec l'appui de 7 régiments d'artillerie de campagne

${ }^{3}$ Archives de l'auteur [Heikant], 14 mars 1945.

4 Ibid.

5 Walter Lüdde-Neurath, Les derniers jours du troisième Reich, traduit de l'allemand par René Jouan (Paris, Berger-Levrault, 1950), 21.

6 Journal de guerre du Régiment, XXXVI: 6.

7 Ibid., 7. 
et 4 régiments d'artillerie moyenne. "C'était un véritable enfer" note le journal de guerre du Essex Scottish ${ }^{8}$. Le même jour, en Allemagne, le brillant ministre des munitions, Speer, écrivait à Hitler pour lui dire que la guerre était perdue ${ }^{9}$.

Le lendemain, le brigadier Leggat et le capitaine Findlater venaient annoncer au Régiment qu'il s'était mérité trois décorations polonaises, décernées respectivement au major Tremblay, au capitaine Sévigny et au lieutenant Caron. Cette joie fut partagée ce soir-là par le capitaine Aimé DesRosiers, arrivé soudainement en visite au Régiment, après un séjour de deux ans en Italie ${ }^{10}$.

Vers cette même date, on pouvait lire dans les journaux de langue française au Canada, sous la plume du correspondant de guerre Phil Lauzon, les déclarations suivantes à propos du Régiment:

L'un des plus fameux régiments d'artillerie [de l'Armée canadienne]... est entièrement composé de Canadiens français; c'est le $4^{\mathrm{e}}$ Régiment d'artillerie moyenne, qui, depuis le jour du débarquement en Normandie, s'est toujours fait remarquer par la précision de son tir ${ }^{11}$.

\section{OPÉRATION "PLUNDER"}

Le 21 mars, le commandant du Régiment réunissait les majors et les capitaines afin de les mettre au courant de la prochaine opération, soit l'offensive sur le Rhin ${ }^{12}$. Dès le lendemain, dans la nuit, le Régiment partait pour sa prochaine destination. Dans l'intervalle, "Brigitte" avait trouvé le moyen de faire un nouveau mauvais coup, comme en témoigne cette phrase d'une lettre d'un officier, ce jour-là ! ..."Brigitte est encore

\footnotetext{
${ }^{8}$ Colonel C. P. Stacey, Histoire officielle de la participation de l'armée canadienne à la seconde guerre mondiale: la campagne de la victoire: les opérations dans le nord-ouest de l'Europe (1944-1945) (Ottawa, Imprimeur de la Reine, 1960), 548.

9 Georges Blond, L'agonie de l'Allemagne (Ottawa, Cercle du Livre de France, 1953), 218.

10 Journal de guerre du Régiment, XXXVI, 7.

11 Archives Dupuis, mars 1945, Mon Album de guerre 1939-1945.

12 Journal de guerre du Régiment, XXXVI: 7.
} 
enceinte, malgré l'ordre formel: “This is Germany, don't fraternize ${ }^{13 "}$.

Voici comment le général Eisenhower devait expliquer plus tard le rôle de l'Armée canadienne dans l'opération "Plunder", c'est-à-dire celle qui allait assurer le franchissement du Rhin:

Sur le flanc gauche, l'Armée canadienne ne devait pas participer à l'assaut d'une façon effective, mais elle tiendrait fermement la ligne du Rhin et de la Meuse, depuis l'ouest d'Emmerich jusqu'à la mer, pour assurer la sécurité absolue de la tête de pont que nous possédions déjà sur le Rhin à Nimègue et couvrir l'estuaire de l'Escaut, le port d'Anvers étant le seul point en arrière de notre front où l'ennemi aurait pu nous gêner effectivement. La $2^{\mathrm{e}}$ armée [britannique] ayant réussi à établir une tête de pont, le $2^{\mathrm{e}}$ corps canadien franchirait le Rhin à Rees et s'emparerait d'Emmerich, où l'Armée canadienne commencerait alors la construction d'un autre pont ${ }^{14}$.

L'opération "Plunder" commençait à 9 heures du soir, le 23 mars, après de puissants bombardements de l'aviation et de l'artillerie ${ }^{15}$. En effet, comme l'affirmait Eisenhower par la suite:

L'offensive fut précédée le 23 mars, à 20 heures, d'un vigoureux barrage d'artillerie de soixante minutes pour neutraliser la rive droite du Rhin et la zone où nos forces aéroportées devaient atterrir le lendemain... Grâce à la puissance de nos tirs préparatoires d'artillerie... nous ne rencontrâmes, d'une façon générale, qu'une légère opposition dans la traversée du fleuve et nous pûmes nous établir fermement sur la rive opposée ${ }^{16}$.

\section{LE GENERAL CRERAR FELICITE LE MAJOR TREMBLAY}

Ce même 23 mars, alors que le Régiment ajoutait la voix tonnante de ses seize pièces au tintamarre final, espérait-on,

${ }^{13}$ Archives de l'auteur [Hasselt], 22 mars 1945.

14 Général Eisenhower, Les opérations en Europe des Forces expéditionnaires alliées ( 6 juin 1944 - 8 mai 1945), traduit par le capitaine Ramsay (Paris, Charles Lavauzelle \& Cie, 1948), 231-232.

15 Stacey, op. cit., 566.

16 Eisenhower, op. cit., 237. 
sur le Rhin, arrivait le message suivant du général Crerar, commandant de l'Armée canadienne, à l'adresse du major Tremblay:

I wish to be among the first, if not the first, to congratulate you on the award of the Distinguished Service Order, made to you by H.M. the King, in recognition of the Distinguished service you have rendered the Canadian Army ${ }^{17}$.

\section{LE REGIMENT EN PLEINE ACTION}

A la fin de la journée du 24 mars, 4,000 prisonniers étaient capturés sur le front anglo-canadien. Le Régiment, pour sa part, se faisait bombarder par quelques avions ennemis qui, heureusement, ne causèrent aucun dommage ${ }^{18}$. Le lendemain, toute la rive gauche du Rhin était nettoyée de troupes allemandes ${ }^{19}$, et l'on annonçait que le général Patton, pour sa part, avait déjà progressé de 30 milles à l'est du Rhin, pour ne s'arrêter qu'à 10 milles au sud-est de Francfort ${ }^{20}$.

Le 28 mars, le Régiment devenait très actif, tirant 2,000

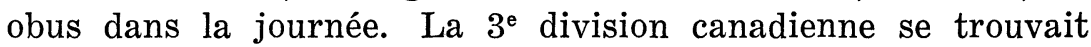
alors rendue aux abords d'Emmerich, et la $2^{\text {e }}$ division canadienne traversait le fleuve à son tour pour prendre position le long de la $3^{\mathrm{e}}$ division ${ }^{21}$.

Le 29 mars, alors que Patton approchait de Nüremberg et que des chars britanniques approchaient de Münster, le Régiment tirait 2,300 obus au-delà du Rhin ${ }^{22}$.

\section{HOMMAGE AU LIEUT. GUI-L. CARON}

Ce même jour, on pouvait lire dans les journaux de langue française au Canada, sous la plume du correspondant de guerre

17 Journal de guerre du Régiment, XXXVI : 8.

18 Ibid., 9.

19 Major M. Shulman, La défaite allemande à l'Ousst, traduit de l'anglais par le capitaine de corvette André Cogniet (Paris, Payot, 1948), 324.

20 Journal de guerre du Régiment, XXXVI : 9-10.

21 Ibid., 10-11.

22 Ibid., 11. 
Jean-Charles Daoust, le texte suivant à propos du lieut. Gui Caron:

Le lt. Gui-Louis Caron, héros montréalais de 23 ans, qui recevra ces jours-ci la plus haute décoration de la Pologne... est remarquable pour sa modestie. La citation officielle publiée par le quartier général de la Défense nationale à Ottawa se passe de commentaires.

Un jeune homme blond, au teint rose, à la moustache blonde, de taille moyenne, le lieutenant Caron s'annonce plutôt à cause de ses verres, comme le genre studieux. Ce n'est pas un colosse comme Dollard Ménard, héros des Fusiliers Mont-Royal à Dieppe. Ce n'est pas l'athlète et l'homme de fer qu'est Paul Triquet, héros du Royal 22 ${ }^{\mathrm{e}}$ Régiment à CasaBerardi. Ce n'est pas non plus comme nos plus récents décorés du D.S.O.: les colonels Mathieu et Smith, des militaires dans la force de l'âge; les majors Ostiguy, Gauvin et Sévigny, des fantassins à l'allure de coureurs de bois. C'est l'étudiant en sciences économiques et politiques de l'université McGill, qui a hâte de retourner à ses cours...

"Comment ai-je mérité cette médaille ?... J'étais dans un gros tank avec mes signaleurs, les canonniers Marcel Boucher, de Cornwall, et Bill Whatmore. Nos compagnons étaient un brave sergent et le chauffeur du tank, tous deux Polonais. Pendant cinq jours et cinq nuits, nous eûmes de nombreuses émotions, pour dire le moins. Le major Maciejowski, le commandant, fut tué le premier jour. Près des trois-quarts des tanks polonais de notre régiment de reconnaissance furent détruits. Le dernier jour, par exemple, le Cromwell qui nous précédait, et le petit tank qui suivait notre gros Sherman éclatèrent en flammes. Evidemment, nous fûmes veinards... La bataille de Falaise fut ma pire expérience", conclut le lieutenant Caron ${ }^{23}$.

Et, le 30 mars, la compagnie Canadian News Reel venait filmer les positions des canons du Régiment, en vue d'une production en couleurs de Warner Bros ${ }^{24}$.

${ }^{23}$ Archives de Jean-Charles Daoust, 29 mars 1945.

24 Journal de guerre du Régiment, XXXVI: 11. 
Le lendemain, le major Tremblay quittait le Régiment pour devenir commandant en second du $1^{\text {er }}$ régiment d'artillerie moyenne. On apprenait, ce même jour, que le Régiment traverserait le Rhin incessamment ${ }^{25}$.

\section{LE REGIMENT AU.DELÃ DU RHIN}

En effet, dès le $1^{\text {er }}$ avril, le Régiment était au-delà du Rhin, ayant traversé sur le pont Blackfriars, près de Rees ${ }^{26}$, où l'artillerie avait joué un rôle essentiel, comme en témoigne ce passage du colonel Stacey:

...Les parachutistes, qui défendaient la ville [Rees], se battirent furieusement, mais l'assaut fut mené résolument en terrain découvert, six régiments d'artillerie de campagne, deux d'artillerie moyenne et deux batteries de 7.2 pouces leur apportant un concours précieux. Dans la ville, l'ennemi opposait encore une résistance désespérée dans des maisons fortifiées; il fallut, pour les réduire, avoir recours aux lance-flammes "Wasp" et à des concentrations de feu d'artillerie ${ }^{27}$.

Le 2 avril, un officier du Régiment écrivait:

A 9 heures, le jour de Pâques au matin, nous traversions le Rhin ... Rien ne peut plus nous arrêter; des milliers de camions, tanks, canons et tout le bazar déferlent en flot continu sur une Allemagne déjà dévastée par l'aviation ${ }^{28}$.

\section{LES JOURNAUX PARLENT ENCORE DU RÉGIMENT}

Pendant que le Régiment se joignait à cette dernière avance en Allemagne, on pouvait lire, le 4 avril, dans les journaux de langue française du Canada, sous la plume du correspondant de guerre Jean-Charles Daoust:

25 Ibid.

26 Lieut.-col. J.-H.-R. Gagnon, O.B.E., et lieuts P.-L. Côté, J.-R. Gouin et P.-M. Pelletier, The History of the 4th Canadian Medium Regt $R C A$ (Hollande, 1945), 15.

27 Stacey, op. cit., 569.

28 Archives de l'auteur [Vorden], le 2 avril 1945. 
Les artilleurs canadiens-français du fameux $4^{\mathrm{e}}$ Régiment d'artillerie moyenne et, plus particulièrement, ceux de la $58^{\circ}$ batterie, ont dû tressaillir d'un orgueil bien légitime depuis quelques jours. Leur courage et leur mépris du danger, fondés sur une compétence reconnue de tous, viennent d'être officiellement proclamés.

Aujourd'hui même, dans un hôpital général militaire canadien en Angleterre, plusieurs héros canadiens et polonais honoraient de leur présence l'investiture du capitaine Pierre Sévigny, 27 ans, de Québec, et du lieutenant Gui-Louis Caron, 23 ans, de Montréal...

Décoré le 17 mars du "Distinguished Service Order", leur commandant âgé de 35 ans, reçoit avec le lieutenant Caron, la Croix de bravoure ...

De plus... le capitaine Luc Chabot, de Kingston, qui commandait l'autre troupe de la batterie du major Tremblay, est cité pour sa bravoure, de même que son sergent-major Edgar Tremblay, de St-Josephd'Alma, Lac St-Jean; le sergent Edgar Mercier, de Mont-St-Pierre; les canonniers Pierre Plante, de Gaspé, Harold Duplessea, de St-Stephens (N.-B.), et André-Jacques Guillotte, de Montréal ${ }^{29}$.

\section{INVESTITURE DU CAPITAINE SÉVIGNY \\ ET DU LIEUTENANT CARON}

Et, le 6 avril, du même correspondant de guerre, on pouvait lire les lignes suivantes, à propos de cette investiture:

En polonais, en anglais et en français, le général Josef Haller, de la $1^{\text {re }}$ division blindée polonaise, a déclaré:

Capitaine Sévigny,

En reconnaissance de votre conduite en face de l'ennemi, au cours des batailles livrées en France par la $1^{\text {re }}$ division blindée polonaise en 1944, le Président de la République polonaise vous décerne la Croix d'argent de l'Ordre Militaire Polonais du

${ }^{29}$ Archives de Jean-Charles Daoust, 4 avril 1945. 
Virtuti Militari, selon l'article de la loi décrétée le $25^{\mathrm{e}}$ jours de mars 1935.

Le capitaine Pierre Sévigny, officier observateur $\mathrm{du} 4^{\mathrm{e}}$ Régiment canadien d'artillerie moyenne, attaché aux escadrons de reconnaissance de la $10^{\mathrm{e}}$ brigade de cavalerie blindée, dirigea le tir de barrage de son Régiment avec un courage admirable, sans tenir compte de la cible qu'il offrait à l'ennemi.

Sur la colline 262, appelée "Maczuga" (Masse), le capitaine Sévigny vit ses communications ordinaires détruites par l'action de l'ennemi, pendant 36 heures. Le capitaine continua quand même à diriger par sans fil le tir de son Régiment. Il servait alors dans les secteurs les plus exposés du front.

Le capitaine Sévigny détruisit des formations de tanks allemands qui attaquaient cette colline 262 en communiquant à ses commandants de batterie (calibre 5.5), alors que lui-même n'était qu'à 300 verges des Panzers, la position exacte de l'ennemi.

Le capitaine Sévigny, par son courage, sa bravoure, son habileté militaire, est considéré comme l'un des principaux défenseurs de la colline "Maczuga" (Masse).

\section{Lieutenant Caron,}

Afin de reconnaître votre courage et votre bravoure, face à l'ennemi, durant la libération de la France en 1944, le Président de la République polonaise vous confère la Croix de bravoure pour la première fois, selon l'article 5 de l'arrêté ministériel de la Défense nationale, décrété le $11^{\mathrm{e}}$ jour d'août 1920 .

Le lieutenant Gui-Louis Caron, officier du $4^{\mathrm{e}}$ Régiment canadien d'artillerie moyenne, a fait preuve de grande bravoure à son poste d'officier observateur de son Régiment, alors qu'il servait sur la première ligne de feu.

Dans son tank d'observation, le lieutenant Caron méprisa tout danger pour permettre aux batteries du $4^{\text {e }}$ Régiment d'artillerie moyenne de protéger de son tir les troupes d'assaut polonaises. 
Sous le feu direct des tanks allemands, le lieutenant Caron, au risque de sa vie, dirigea avec un sangfroid remarquable le tir de barrage nécessaire à la traversée de la rivière Dives, dans le secteur de Jort, par le $10^{\mathrm{e}}$ détachement de reconnaissance polonais.

Dans cette dernière action, le lieutenant Caron, sans avoir reçu d'ordres officiels à cette fin, répondit spontanément à l'appel du danger qui menaçait le $10^{\mathrm{e}}$ détachement de reconnaissance polonais.

Ainsi, cet officier fit plus que son devoir ${ }^{30}$.

\section{ELOQUENCE DES DOCUMENTS}

Malgré la longueur de ces documents, j'ai cru bon de les citer au complet, car ils font partie intégrante de l'histoire du Régiment, et méritent de passer à la postérité. D'ailleurs, à compter de maintenant, j'estime de mon devoir d'historien de laisser parler de plus en plus les documents eux-mêmes, qui sont beaucoup plus éloquents que tout ce que je pourrais écrire. Dès son retour au Canada, le capitaine Sévigny déclarait à un journaliste de La Presse.

Il y a sûrement négligence coupable, et je ne crois pas que l'expression soit trop forte, de la part de certains de nos dirigeants qui ne proclament pas assez haut les faits d'armes des troupes canadiennesfrançaises sur les champs de bataille de France, de Belgique, de Hollande et d'Allemagne... Ne sait-on donc pas tout ce que les Canadiens français ont fait ?

... Cette unité de 800 hommes [le $4^{\mathrm{e}}$ Régiment], tous Canadiens français, s'est battue sans arrêt, sans repos, du 6 juillet jusqu'à ce jour. Elle se bat encore en Allemagne. L'unité a perdu 40 p. 100 de son effectif, et pas moins de 4,000 hommes, tous Canadiens français, y ont été incorporés pour remplacer les pertes ${ }^{31}$.

30 Ibid., 6 avril 1945.

31 Archives Dupuis. 
Sans doute l'appel du capitaine Sévigny fut-il entendu, puisque, dans un journal de langue anglaise de Montréal, on pouvait lire, quelques jours plus tard:

The story of the closing of the Falaise gap, which last summer enabled Allied armies to thrust forward across France and Belgium to the heart of Germany is the story of Hill 262. And it is an epic story, - of bloodshed, determination and superb gallantry of French-Canadian and Polish troops ${ }^{32}$.

\section{MALGRE LES ÉLOGES, LE REGIMENT CONTINUE À COMBATTRE}

Pendant que tous ces éloges étaient attribués au Régiment, celui-ci n'en continuait pas moins à combattre et à talonner l'ennemi. Le 6 avril, il était encore en route, s'arrêtant pour tirer sur l'ennemi en fuite, puis reprenant sans arrêt sa course. Malgré les inquiétudes du capitaine Sévigny sur le peu de réclame faite autour du Régiment, ce dernier n'en était pas moins à l'honneur au Canada même, comme en témoigne ce passage d'une lettre d'un officier du Régiment, en date du 7 avril:

Je suis content de constater que la réputation du $4^{\mathrm{e}}$ Médé, comme tu dis, n'est plus à faire... C'est avec un orgueil très légitime que je puis me considérer comme un vieux de la vieille dans ce Régiment, car nous avons déjà plusieurs exploits à notre crédit. Dernièrement encore, nous avons contribué à faire tomber Emmerich, un des bastions du Rhin ${ }^{33}$.

\section{SOUVENIR DE GRAYSHOTT}

Les membres du Régiment qui, pendant leur séjour à Grayshott, en Angleterre, ont connu l'hospitalité de M. et $\mathbf{M}^{\text {me }}$ Robert Spake, ne liront pas sans émotion ces extraits d'une lettre de $\mathbf{M}^{\mathrm{me}}$ Spake, en date du 7 avril 1945:

Voilà bien longtemps que nous avons eu le plaisir de vous lire. Naturellement, je présume que vous n'avez guère le temps de faire de la prose, et que

32 Ibid.

33 Archives de l'auteur [Laren], 7 avril 1945. 
vous devez passer par bien des situations pénibles et hasardeuses. Heureusement que la fin est en vue, et vous tous là-bas n'aurez pas volé de retrouver famille et repos ... Ici, la maison est toujours fréquentée par les Canadiens français... J'espère que vous passez parfois en revue nos discussions du coin $d u$ foyer; vous devez même parfois y trouver matière à réflexions (si vous avez le temps de réfléchir). Avez-vous parfois rencontré Bélanger ?... $\mathrm{Si}$ vous venez en Angleterre, n'oubliez pas la maison ... Je dois vous dire que je suis toujours occupée à faire les petites tartes à la confiture que vous connaissez bien, car je dois ravitailler les amis canadiens qui viennent me tenir compagnie et discuter.... ${ }^{34}$.

\section{LE RÉGIMENT DE NOUVEAU AVEC LES POLONAIS}

Le 8 avril, le Régiment était fort occupé à tirer sur des pelotons ennemis. Dans la soirée, on apprenait que le Régiment serait de nouveau attaché aux Polonais. "La rumeur circule que les Polonais ne veulent pas combattre à moins d'être appuyés par le $4^{\mathrm{e}}$ Régiment ${ }^{35}$." Dès le lendemain, le lieut. Houle se rendait comme officier de liaison auprès des Polonais. Avec ces derniers, une autre opération de "balayage" allait se dérouler:

Tu emploies une expression juste en écrivant le mot "balai" au sujet de nos opérations actuelles. En effet, l'ennemi ne présente plus aucune résistance sérieuse, sauf dans la Rhur, où, comme des rats pris au piège, quelques fanatiques isolés frétillent encore. On rapporte même que certains parachutistes de la fameuse Hitler Jugend se sont laissés brûler vif par les lance-flammes plutôt que de se rendre. Tu vois où mènent les doctrines néfastes du nazisme ${ }^{36}$.

\section{CIVILS ALLEMANDS}

Et cette opération "balayage" n'était pas sans bousculer brusquement les quelques civils allemands restés sur la voie de l'invasion, comme en témoigne cet extrait de lettre:

${ }^{34}$ Ibid.

35 Journal de guerre du Régiment, XXXVII: 3.

36 Archives de l'auteur [Laren], 9 avril 1945. 
J'ai dû surmonter avec un grand effort, hier, mon penchant pour la pitié envers les gens. Voici ce qui s'est passé: le major Archer me donna instruction de trouver des quartiers pour passer la nuit. Je suis parti en avant du Régiment, en jeep. Voyant deux belles grandes fermes, je décidai à l'instant que nous arrêterions là pour la nuit.

Je m'approchai lentement d'une de ces fermes. Pas une âme ne semblait bouger... Tout le monde semblait séché de frayeur. Car les Allemands, conscients des atrocités qu'ils ont commises en Russie et ailleurs, craignent les représailles. Heureusement que nous sommes plus civilisés que leurs troupes d'élite. J'ouvre la porte, et une vieille femme, tremblotant de frayeur, me regarde avec des yeux désespérés. Sachant qu'il faut être sévère... j'ai dit tout simplement avec assez de fermeté, quoique déjà la pitié m'envahissait: "Soldaten slapen hier, abend." Elle comprit mon jargon. Je me dirigeai vers l'autre ferme où la même scène se produisit. Là, j'ai installé le mess des officiers. Le père, la mère et le fils rampaient comme des chiens battus ... Là où j'ai failli céder à la pitié fut lorsque je suis allé à une troisième maison où je voulais installer notre médecin. Il n'y avait là que le père, la mère et la fille. Tous les trois esquissèrent d'abord un vague mouvement de fuite à travers les bois. Mais, voyant que je n'étais pas si menaçant, ils s'éloignaient lentement tout en me regardant. Je fis un signe à la mère, dont la seule faute fut de fournir trois fils à l'armée allemande. J'entrai avec elle dans la maison, où flottaient la mort et la désolation. En sortant, la fille nous avait rejoints. En sanglotant... elle me fit comprendre que ses trois frères étaient morts à la guerre. A ces mots, la mère éclata en sanglots. J'ai fait un geste, moitié sympathique moitié pour leur montrer qu'eux seuls étaient responsables de leur malheur. J'ai quitté cette scène l'amertume dans l'âme ${ }^{37}$.

\section{HOMMAGE AU "PADRE" LUCIEN CLERMONT}

A partir du 12 avril, on peut dire que la guerre tirait vraiment à sa fin, du moins pour le Régiment. En effet, les Polonais

37 Ibid. [Striepe], 10 avril 1945. 
passaient surtout leur temps à construire des ponts pour permettre au Régiment d'avancer, mais il n'y avait presque plus de tir. Le 13 avril, on organisait une partie de soft-ball entre les officiers et le personnel du Q.G. régimentaire, ce qui est significatif du ralentissement du combat ${ }^{38}$. Le même jour, on apprenait que le "padre" venait de recevoir la Croix de Guerre avec étoile d'or des autorités françaises, pour sa bravoure autour de Caen ${ }^{39}$. A ce propos, voici le texte qu'on pouvait lire dans La Presse du 30 juin suivant, reproduit de la Semaine religieuse:

$\mathrm{Au}$ cours d'un raid aérien sur le faubourg de Vaucelles, dans la nuit du 27 juillet 1944, pendant que les obus éclataient près des canons, le capitaine honoraire Clermont se précipita vers le secteur de tir, s'empressa auprès des mourants et des blessés, et aida à les évacuer, tandis que la région était encore bombardée. Quoique cinq pièces d'artillerie aient été mises hors de service par les avions ennemis et que le secteur de tir ait encore été soumis à un bombardement formidable, le capitaine Clermont passa le reste de la nuit à se rendre d'un canon à un autre pour encourager les hommes qui manœuvraient les batteries anti-avions.

Le 30 juillet 1944, au faubourg de Vaucelles, un dépôt de munitions pour pièces de 25 livres situé immédiatement en arrière du poste de tir fut frappé par un obus ennemi. Les munitions prirent feu et les obus commencèrent à éclater; un homme fut tué et plusieurs furent blessés. Le capitaine Clermont, qui se trouvait dans cette région, et s'entretenait avec les servants des pièces, se rendit immédiatement au dépôt des munitions encore sous le feu de l'ennemi et sauva trois hommes au risque de sa propre vie.

Le 18 août 1944, près de Les Moutiers-en-Auge, tandis que le Régiment soutenait une division blindée polonaise, l'ennemi mit le feu à quelques véhicules de munitions. Plusieurs militaires venaient de tomber et tous les préposés aux premiers soins s'empressaient auprès des blessés. Le capitaine Clermont s'offrit à évacuer un homme qui réclamait des

38 Journal de guerre du Régiment, XXXVII : 5.

39 Archives de l'auteur [Sleen], 13 avril 1945. 
soins urgents. Les véhicules en flammes encombraient la seule issue et, au mépris des avertissements de la prévôté, que le passage était dangereux, le capitaine Clermont traversa ce brasier où les munitions sautaient et réussit à évacuer le blessé ${ }^{40}$.

\section{LIBERATION DE PRISONNIÈRES POLONAISES}

Le 14 avril, les Polonais, toujours soutenus par le Régiment, libéraient d'un camp de concentration 1,700 Polonaises capturées l'année précédente pendant le soulèvement de Varsovie. Un colonel polonais, pour sa part, était heureux d'y retrouver sa femme et sa fille. Malgré cette joie délirante des Polonais, la poursuite de l'ennemi ne ralentissait pas, puisque le même jour le Régiment avançait encore de 10 milles ${ }^{41}$.

\section{ARROGANCE DU DÉSESPOIR}

Pendant que les troupes allemandes s'effondraient sur tous les fronts, Hitler osait encore affirmer, le 14 avril 1945:

Ce sont seulement les étrangers qui arborent des drapeaux blancs et non les Allemands; ceux-ci en sont incapables; tout le peuple allemand n'a aujourd'hui, unanimement, qu'une seule pensée: se battre jusqu'au dernier homme contre l'ennemi qui veut nous exterminer ${ }^{42}$.

\section{LE RÉGIMENT DÉTRUIT DES CHARS ENNEMIS}

Quelle illusion! Pourtant, sur le front du Régiment, tout au moins, il y avait encore à faire, car le 15 avril il lui fallait encore avancer afin d'engager l'ennemi retranché sur la rive nord du canal de Küsten. Dès le 16, le Régiment tirait 20 obus par canon, et le 17, grâce au lieut. Martin, officier observateur, le Régiment détruisait deux chars ennemis ${ }^{43}$. Le lendemain, il détruisait un canon ennemi. Le 19, l'opération sur le canal Küsten était réussie, et plus de 400 prisonniers étaient capturés.

\footnotetext{
${ }^{40}$ Archives Dupuis.

41 Journal de guerre du Régiment, XXXVII: 6.

42 Général Karl Koller, Le dernier mois (14 avril - 27 mai 1945), traduit de l'allemand par René Jouan (Paris, Payot, 1950), 11. 43 Journal de guerre du Régiment, XXXVII : 7.
} 
On rapportait en outre que le tir du Régiment avait été très efficace. Ainsi, à une occasion, appelé à briser une contreattaque, le Régiment provoqua une levée en masse de drapeaux blancs ${ }^{44}$. Le 20 avril, le Régiment traversait le canal de Küsten et s'installait au nord de Lehe, après une nouvelle avance de 8 milles. La résistance s'écroulait de toutes parts, et les prisonniers affluaient, alors que, le long des routes, les maisons étaient toutes en flammes ${ }^{45}$. Il n'est pas étonnant qu'à cette date, dans son bunker à Berlin, Hitler “... physiquement, donnait l'impression d'être brisé, bouffi, voûté, épuisé et nerveux ${ }^{46}$."

\section{AVANCE DE 135 MILLES}

Le 22 avril, le Régiment avait franchi 135 milles depuis qu'il était attaché aux Polonais, et se dirigeait désormais sur un axe nord-est en direction de Wilhemshaven. Mais il fallait encore construire trois ponts avant d'atteindre cette destination ${ }^{47}$. Ce même jour, un officier du Régiment donnait ses impressions de la récente avance:

Depuis quelques jours, nous poursuivons l'ennemi sans répit... Les vieilles scènes de Normandie se reproduisent dans toute leur horreur: des cadavres, des ruines et des prisonniers hagards. Toutes les maisons sont en feu, et les familles allemandes subissent le châtiment qu'elles se sont attiré, errent pêlemêle sur les routes... Nous assistons, muets et saisis, à la mort d'une nation, celle qui prétendait dominer toutes les autres. Je ne sais pas combien d'années il faudra pour réorganiser ce chaos: c'est indescriptible. Des prisonniers français, belges et russes libérés par notre avance, ajoutent au problème déjà compliqué du transport, du ravitaillement, etc. L'Europe subit en ce moment les mêmes angoisses qui ont suivi l'invasion des Barbares au moyen âge. Comme nous sommes heureux, au Canada, d'être loin de tout cela ${ }^{48}$.

${ }^{44} \mathrm{Ib} i d ., 8$.

45 Ibid., 8-9.

46 Neurath, op. cit., 29.

47 Journal de guerre du Régiment, XXXVII: 9.

48 Archives de l'auteur [Flashmeer], 22 avril 1945. 


\section{HITLER EST EFFONDRE}

Ce même jour également, un général allemand de l'entourage de Hitler rapportait ce qui suit:

Le Führer est complètement effondré, il se rend compte désormais que la lutte est sans espoir... L'ambiance, dans l'abri, est épouvantablement déprimante, il m'est impossible d'en donner une idée exacte. Dans l'entourage du Führer, les subalternes boivent pour se donner du courage, les femmes et les jeunes filles aussi ${ }^{49}$.

\section{PRÉVISIONS D'APRĖS-GUERRE}

Le 23 avril, le Régiment tirait encore 40 obus par canon. Déjà, toutefois, on commençait à penser non seulement à la fin de la guerre, mais à la tâche qui attendrait les armées après cette longue épreuve. Voici ce qu'écrivait, à ce propos, un officier du Régiment le 24 avril:

Le colonel profita d'une telle réunion d'officiers... pour soulever quelques points sur la discipline en général et, en particulier, sur l'avenir immédiat qui nous attend concernant l'occupation de l'Allemagne. Le problème est complexe et formidable; on ne peut se faire une idée du chaos, tant que l'on ne patauge pas dedans. L'armée, de concert avec les hommes d'Etat, les économistes, les sociologues et, en somme, de concert avec tous les hommes de bonne volonté de la terre entière, ont tous ensemble une tâche énorme à entreprendre. Il s'agit de remettre sur pied une civilisation agonisante...

Nous faisons des prisonniers tous les jours; des enfants de seize ans, habillés en soldat, viennent nous prier de les faire prisonniers, car ils sont sans nourriture, sans but, sans rien du tout. Quand nous leur parlons du Führer, c'est comme si nous leur parlions d'un fléau ...

$\mathrm{Au}$ moment où j'écris ces lignes, les Russes se battent au cœur de Berlin ${ }^{50}$.

49 Koller, op. cit., 32.

50 Archives de l'auteur [Flashmeer], 24 avril 1945. 


\section{LE REGIMENT MITRAILLÉ PAR L'AVIATION ALLEMANDE}

Le 26 avril, le Régiment tirait encore 53 obus par canon dans la journée, pour appuyer une attaque destinée à établir une tête de pont au nord de la Leda. Ce soir-là, encore, un avion ennemi venait mitrailler les positions du Régiment ${ }^{51}$.

\section{JONCTION DES AMÉRICAINS ET DES RUSSES}

La grande nouvelle du 27 avril était la jonction des Américains et des Russes à Torgau, sur l'Oder. Voici ce qu'écrivait un officier du Régiment, le surlendemain:

... Les événements se précipitent plus que jamais, comme dans une espèce de vertige. Certes, nous sommes encore en Allemagne, et nos canons menacent toujours l'ennemi, mais pour nous la guerre est virtuellement terminée. Il n'y a plus de résistance nulle part... Nous écoutons les nouvelles, heure après heure, et l'optimisme est dans l'air ${ }^{52}$.

\section{DISCIPLINE}

Le 4 mai, dans les Ordres du jour, partie II, du Régiment, on pouvait lire ce texte rédigé par le commandant:

Tous les Canadiens doivent se comporter de manière à maintenir leur bonne réputation. Pour citer les mots de notre général: "Le prestige militaire des Canadiens n'a jamais été aussi élevé. Tout officier ou soldat se doit à lui-même et aux milliers de ses camarades tombés au champ d'honneur, de se bien comporter et de se bien conduire, comme un militaire authentique de premier ordre, telle que sa réputation, gagnée durant la bataille, l'indique si clairement."

Les Canadiens doivent se bien conduire, et devront le faire en tout temps. Et, en ce qui nous concerne dans le Régiment, nous devons nous efforcer de faire ce que nous avons toujours fait dans le passé avec succès. D'autres régiments peuvent être aussi bons que le nôtre, mais nul ne doit être meilleur ${ }^{53}$.

51 Journal de guerre du Régiment, XXXVII : 12.

52 Archives de l'auteur [Kollinghorst], 29 avril 1945.

53 Archives Gagnon. 


\section{MORT DE HITLER}

Le 2 mai, on apprenait la grande nouvelle de la mort de Hitler, puis la reddition sans conditions des armées allemandes en Italie $(600,000$ à 900,000 prisonniers). Enfin, la capitulation de Berlin, où 7,000 hommes avaient abandonné le combat ${ }^{54}$. Le lendemain, Hambourg, Kiel et Prague étaient déclarées villes ouvertes et, partout en Europe, les Allemands étaient prêts à capituler, ce qu'ils firent d'ailleurs dès le 4 mai. Le 6 mai, le Régiment avait atteint son point le plus avancé, soit le village de Marx, tout près de Wilhemshaven ${ }^{55}$.

\section{DIFFICULTÉS DE L'AVANCE DU REGIMENT}

Afin de montrer à quel point l'avance du Régiment avait été ardue avec les Polonais, citons ce passage du colonel Stacey:

Le caractère continu de l'avance des Polonais, bien qu'elle fût ralentie par l'état des routes, concourut, dans une certaine mesure, au succès des opérations de la $4^{\mathrm{e}}$ division blindée. Jusqu'à la fin, toutefois, les Polonais continuèrent à rencontrer des résistances... En action jusqu'au bout, l'artillerie polonaise [et le Régiment, bien entendu] ne cessa de pilonner les positions ennemies jusqu'à une minute avant l'ordre de cesser le feu, le matin du 5 mai ${ }^{56}$.

Le Régiment, enfin libéré des soucis de la guerre, allait désormais se préparer à rentrer au Canada, tout couvert de gloire *.

Fin

JACQUES GOUIN,
ex-lieutenant d'artillerie, diplômé en sciences
politiques (Ottawa), correspondant cana-
dien à la Revue d'Histoire de la 2e guerre
mondiale (France), chef adjoint du Bureau
des traductions et rédacteur de l'édition
française du Journal de l'Armée canadienne,
ministère de la Défense nationale, Ottawa.

${ }^{54}$ Journal de guerre du Régiment, XXXVIII : 1-2.

55 Ibid., 3.

56 Stacey, op. cit., 633.

* Cet article est le dernier extrait, à paraître dans la Revue, de notre ouvrage intitulé: Par la bouche de nos canons: Histoire du $4^{e}$ Régiment canadien d'artillerie moyenne (1941-1945), qui sera publié au complet, avec cartes, photos, appendices, index et bibliographie, dans un avenir prochain. 